\title{
Characteristics of primary rat microglia isolated from mixed cultures using two different methods
}

\author{
Li Lin ${ }^{1,2}$, Rakhi Desai ${ }^{2}$, Xiaoying Wang ${ }^{2}$, Eng H. Lo ${ }^{2^{*}}$ and Changhong Xing ${ }^{2^{*}}$ (D)
}

\begin{abstract}
Background: Microglial cultures comprise a critically important model system for investigating inflammatory mechanisms in almost all CNS disorders. Mild trypsinization and shaking are the two most commonly used methods to isolate primary microglia from mixed glial cultures. In this study, we characterized and compared microglia obtained using these two methods.
\end{abstract}

Methods: Primary rat microglia cultures were prepared from cerebral cortices of 1-2-day-old neonatal Sprague-Dawley rats. After achieving confluency at about 14 days in vitro, microglia were isolated from mixed glial cultures via either mild trypsinization or shaking. The purity of microglia was estimated by flow cytometry. Quantitative real-time PCR was used to measure mRNA expression. TNFa, IL-1 $\beta$, IL-10, and IGF-1 in cell culture supernatant were measured using ELISA kits. Phagocytic function was assessed using fluorescein-labeled Escherichia coli K-12 BioParticles.

Results: Mild trypsinization generated a higher yield and purity than shaking. Microglia isolated by mild trypsinization appeared to be in a quiescent state with ramified morphology. Microglia isolated by shaking showed a more heterogenous morphology, including cells with rounded shapes suggestive of activation. Compared with shaking, microglia isolated by trypsinization also had lower baseline phenotype markers (iNOS, CD86, CD206, and arginase 1) and lower levels of cytokines (TNFa, IL-1 $\beta, I L-10$, and IGF-1) as well as reduced phagocytic capability. Both methods yielded microglia that were responsive to various stimuli such as IL-4, lipopolysaccharide (LPS), or interferon- $\gamma$ (IFNY). Although stimulated patterns of gene expression and cytokine release were generally similar, there were also significant differences in terms of absolute response. LPS treatment induced significantly higher levels of TNFa and IL-10 in microglia isolated by mild trypsinization versus shaking. IFNy induced a lower response in TNFa in microglia obtained by mild trypsinization versus shaking.

Conclusions: Our results suggest that isolating microglia with the shaking method may induce slight activation even at baseline, and this may affect stimulus responses in subsequent experiments. Caution and attention should be warranted when choosing isolation protocols for primary microglia cultures.

Keywords: Microglia, Primary culture, Mild trypsinization, Shaking, Phenotype, Immune response

\footnotetext{
* Correspondence: Lo@helix.mgh.harvard.edu;

xing.changhong@mgh.harvard.edu

${ }^{2}$ Neuroprotection Research Laboratory, Departments of Radiology and

Neurology, Massachusetts General Hospital, Harvard Medical School, MGH

East 149-2401, Charlestown, MA 02129, USA

Full list of author information is available at the end of the article
} 


\section{Background}

Microglia are resident immune cells of the brain, constantly monitoring the microenvironment and responding to any kind of pathologic change. One striking feature of microglia is their highly dynamic nature [1]. Microglia can be activated by a large number of stimuli and change in their morphology, cytokine/chemokine expression profiles, and function. Depending on the stimulus and context, activated microglia exhibit a spectrum of phenotypic and functional diversity, ranging from the so-called classically activated (M1-like) to alternatively activated (M2a, M2b, and M2c) [2-9]. M1-like microglia promote the release of various proinflammatory cytokines, thus inducing bystander tissue injury [9-15]. By contrast, M2-like macrophages may actively promote tissue remodeling and repair [9, 12-17].

Primary microglia cultures comprise a useful in vitro tool for exploring a wide range of inflammatory mechanisms in central nervous system (CNS) disease and investigating therapeutic strategies that may target microglia. In vitro, M1-like phenotype is achieved by exposing cells to lipopolysaccharides (LPS) and interferon- $\gamma$ (IFN $\gamma$ ), whereas interleukin (IL)-4 and IL-13 are commonly used to induce M2-like phenotype [18]. However, there are many ways to prepare primary microglia, and some caution may be warranted because these highly reactive cells may respond differently under different isolation conditions. In this study, we isolated microglia from rat brains using two commonly used methods (shaking versus mild trypsinization) and assessed and compared their morphology, gene expression profiles, and cytokine release under baseline and stimulated conditions.

\section{Methods}

\section{Reagents}

DMEM/F12, $0.25 \%$ trypsin-EDTA, and fetal bovine serum (FBS) for cell culture were from Gibco.

Primary antibody against Iba-1 and Alexa Fluor 555conjugated secondary antibody were from Abcam and Molecular Probe, respectively. The antibodies of CD11b, CD45, and CD68 for flow cytometry detection were purchased from $\mathrm{BD}$, eBioscience, and Bio-Rad, respectively, and were diluted according to the manufacturer's instructions. Recombinant rat IL-4 was from Amsbio. LPS was from Sigma-Aldrich. Recombinant rat IFN $\gamma$ was from $R \& D$. The stock solutions of IL-4, LPS, and IFNY were prepared using sterile $d d^{2} \mathrm{H}_{2} \mathrm{O}$, and $d d^{2} \mathrm{H}_{2} \mathrm{O}$ was used as control.

\section{Primary rat microglia culture}

A PubMed search was conducted using the terms "microglia AND (rat OR mouse) AND primary AND culture NOT review [publication type]" from 2006 to 2016. This search resulted in 392 articles. One hundred twenty-five articles were excluded (81 did not use microglial culture, 25 microglia used cell lines, 19 were not available for fulltext access), resulting in 267 articles that used primary cultures of rodent microglia. This quick survey of the literature suggested that two most commonly used methods to purify microglia involved shaking and mild trypsinization (204 used shaking and 26 of them used mild trypsinization) (Fig. 1). Based on this initial analysis, we compared microglia obtained with shaking versus trypsinization. Primary rat microglia cultures were prepared from cerebral cortices of 1-2-day-old neonatal Sprague-Dawley rats. After removing the meninges, the cortical tissues were digested with $0.25 \%$ trypsin-EDTA for $30 \mathrm{~min}$ at $37{ }^{\circ} \mathrm{C}$, followed by mechanical triturating in DMEM/F12 with $10 \%$ fetal bovine serum. The mixed cortical cells were passed through a 70- $\mu \mathrm{m}$ nylon mesh cell strainer and plated on non-coated plastic dishes or plates in DMEM/ F12 with $10 \%$ FBS, and the medium was completely replaced every 3-4 days. After achieving confluency at about 14 days in vitro, the microglia were isolated from mixed glial cultures via either mild trypsinization (enzyme, E) or shaking (S). The mild trypsinization was performed according to previously described methods $[19,20]$. Incubation of mixed glial cultures with a trypsin solution $(0.25 \%$ trypsin-EDTA diluted 1:4 in DMEM/F12) for 15-25 min resulted in the detachment of an intact layer of cells in one piece. Microglial cells remained attached to the bottom of the well. For the shaking method [21, 22], confluent mixed glial cultures were placed on an orbital shaker at $220 \mathrm{rpm}$ for $1 \mathrm{~h}$. The supernatant containing the detached microglial cells was collected and re-seeded for $1 \mathrm{~h}$ to allow microglial attachment. After $1 \mathrm{~h}$, the nonadherent cells were removed. Microglia isolated from both methods were allowed to rest overnight prior to treatments. To compare the yield, we plated the cells from one brain of neonatal pups into one 6-well plate. Yield was calculated as cell numbers per field (six random fields of $\times 200$ magnification per culture, $n=5$ cultures). The cells were fixed in $4 \%$ paraformaldehyde for $30 \mathrm{~min}$, blocked

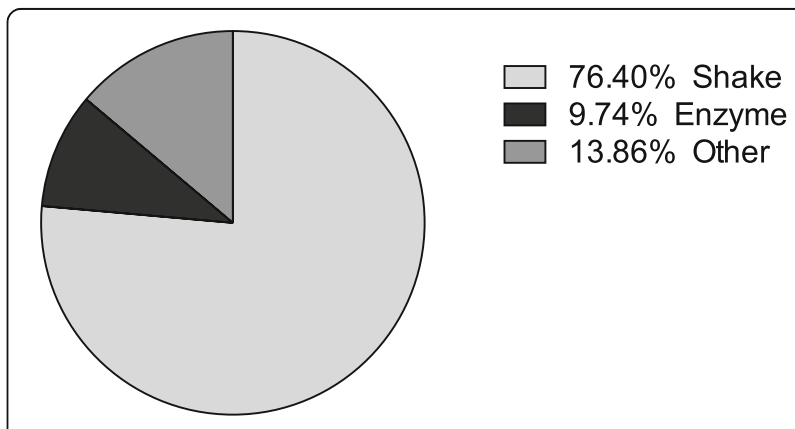

Fig. 1 The proportion of various methods that are used for primary rodent microglial culture by searching PubMed using the term "microglia AND (rat OR mouse) AND primary AND culture NOT review [publication type]" 
with $5 \%$ normal horse serum for $1 \mathrm{~h}$, and incubated with primary antibody against Iba- $1(1: 100)$ at $4{ }^{\circ} \mathrm{C}$ overnight. After washing, the cells were incubated with Alexa Fluor 555-conjugated secondary antibodies (1:200) for $1 \mathrm{~h}$ at room temperature. Negative controls were incubated without primary antibodies, and no immunoreactivity was observed in these controls.

\section{Flow cytometry}

The purity of microglia obtained from mild trypsinization or shaking was estimated by flow cytometry. The cells were collected and labeled with fluorochromeconjugated monoclonal antibodies recognizing antigens (CD11b, CD45, and CD68) at $4{ }^{\circ} \mathrm{C}$ for $15 \mathrm{~min}$. After labeling, the cells were washed twice in PBS and resuspended at a final volume of $400 \mu \mathrm{l}$. Flow cytometry was performed on a BD LSDII, and data were analyzed using FlowJo software.

\section{Real-time PCR}

Quantitative real-time PCR was used to measure mRNA expression. Cultured microglia were treated with IL-4 (20 ng/ml), LPS (50 ng/ml), or IFN $\gamma(20 \mathrm{ng} / \mathrm{ml})$ for $8 \mathrm{~h}$. Total RNAs were extracted using miRNeasy kit (Qiagen) from primary cultured microglia with or without treatment. One hundred nanograms of total RNAs were reverse transcribed into cDNA using $M-M L V$ reverse transcriptase (Invitrogen). Quantitative expression of inducible nitric oxide synthase (iNOS), CD86, CD206, arginase 1, CX3C chemokine receptor 1 (CX3CR1), tolllike receptor 2 (TLR2), and C-C chemokine receptor 2 (CCR2) were measured using gene-specific TaqMan Gene Expression Assays (ABI 7500HT, Applied Biosystems). Relative baseline gene levels were calculated by subtracting $\mathrm{Ct}$ value of $\beta 2$-microglobulin (B2M) from $\mathrm{Ct}$ value of detected genes. Changes in gene expression (fold change) after various treatments were determined using the $2^{-\Delta \Delta C t}$ method with normalization to B2M. All real-time PCRs were performed in triplicates. All experiments were repeated three to six times independently. Activated microglia include a spectrum of various states. The representative genes we selected, including iNOS, CD86, CD206, arginase 1, CX3CR1, TLR2, and CCR2, are closely relevant to different activating states of microglia. To compare the "average fingerprint" of cultured microglia, quantitative data were analyzed using two-way ANOVA $(p<0.05$ for significance, SPSS 21).

\section{ELISA}

Cultured microglia were treated with IL-4 $(20 \mathrm{ng} / \mathrm{ml})$, LPS (50 ng/ml), or IFN $\gamma(20 \mathrm{ng} / \mathrm{ml})$ for $24 \mathrm{~h}$. Tumor necrosis factor $\alpha$ (TNF $\alpha)$ (eBioscience), IL-1 $\beta$ (R\&D), IL-10 (eBioscience), and insulin-like growth factor-1 (IGF-1)
$(R \& D)$ in cell culture supernatant were measured using ELISA kits, according to the manufacturer's instructions.

\section{Microglia phagocytic function assays}

To assess phagocytosis, microglial cells cultured in 6well plates were digested using $0.25 \%$ trypsin-EDTA and re-seeded to a 96-well plate at a concentration of $3.0 \times$ $10^{4}$ cells/well. Then, the cells were incubated with fluorescein-labeled Escherichia coli K-12 BioParticles (Invitrogen) for $2 \mathrm{~h}$ at $37{ }^{\circ} \mathrm{C}$. The cells were rinsed with $0.25 \mathrm{mg} / \mathrm{ml}$ trypan blue to quench extracellular fluorescence. Intracellular fluorescence was read using a fluorescence microplate reader setup with excitation at $480 \mathrm{~nm}$ and emission at $520 \mathrm{~nm}$. The experiments were performed with five replicates per condition and repeated four times.

\section{Statistical analysis}

Data were expressed as mean \pm SE. Three to six separate experiments were performed. Data of real-time PCR were analyzed using two-way ANOVA. Data of ELISA that measures the cytokine release after various treatments were analyzed using one-way ANOVA. Other data were analyzed using $t$ test between two isolation methods. Statistical significance was set at $p<0.05$.

\section{Results \\ Morphology, yield, and purity}

Phase contrast microscopy revealed distinct morphology of microglia isolated using two different methods. Microglia isolated using mild trypsinization showed uniform ramified morphology with short processes and small cell body (Fig. 2a). The morphology of microglia isolated using shaking was more heterogeneous. Most of them showed enlarged round cell body with reduced processes (Fig. 2b). Cells from both methods were Iba-1 positive (Fig. 2c, d). The yield of microglia cultures was higher by using mild trypsinization (76.18 \pm 13.08 cells/field) than by using shaking (54.27 \pm 9.19 cells/field) ( $p=0.015, t$ test) (Fig. 2e).

Besides morphology, the size of microglial cells obtained from the two methods was also different. Measured by flow cytometry with forward scatter (FSC), the average size of microglia isolated using shaking was 1.17-fold larger than those obtained using mild trypsinization ( $p=0.030, t$ test) (Fig. $2 \mathrm{f}-\mathrm{h}$ ). The purity of cultured microglia was determined by flow cytometry with CD11b and CD45 staining. The proportion of CD11b-positive cells (Fig. 2i-k) and CD45-positive cells (Fig. 2l-n) were significantly higher in the mild trypsinization group (93.68 $\pm 2.54 \%$ and $94.92 \pm 3.64 \%)$ compared to the shaking group $(82.9 \pm 7.61 \%$ and $88.08 \pm 3.32 \%) \quad(p=0.028$ for CD11b, $p=0.024$ for CD45, $t$ test). 

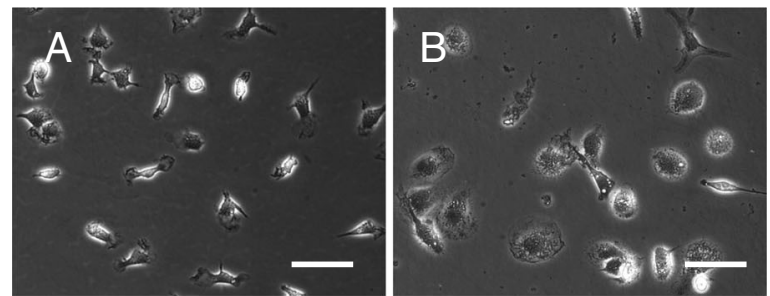

\section{$E$}
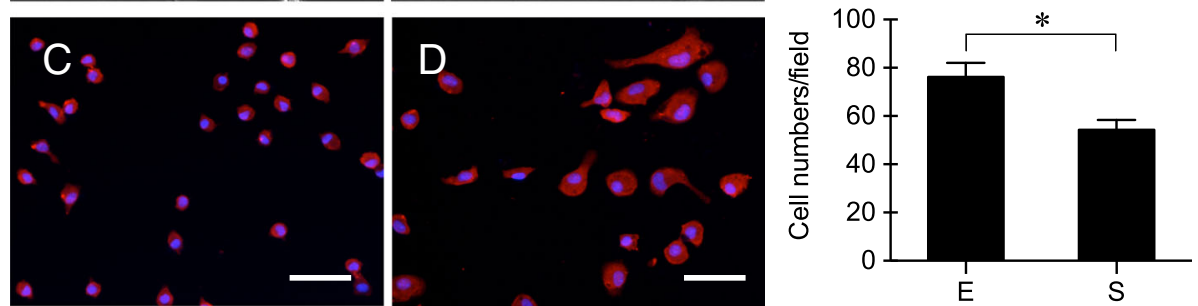

F

G
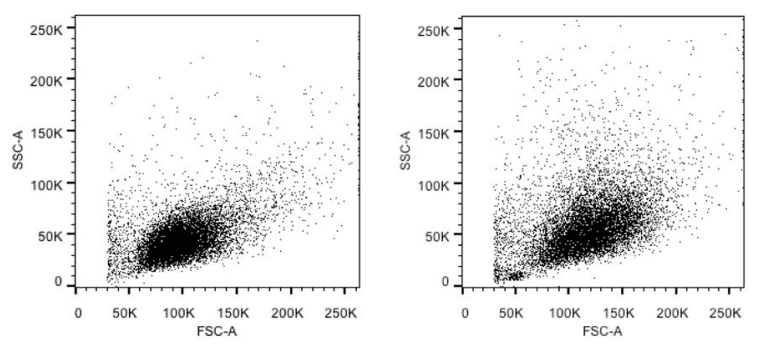

$\mathrm{H}$

$$
\text { I }
$$

J
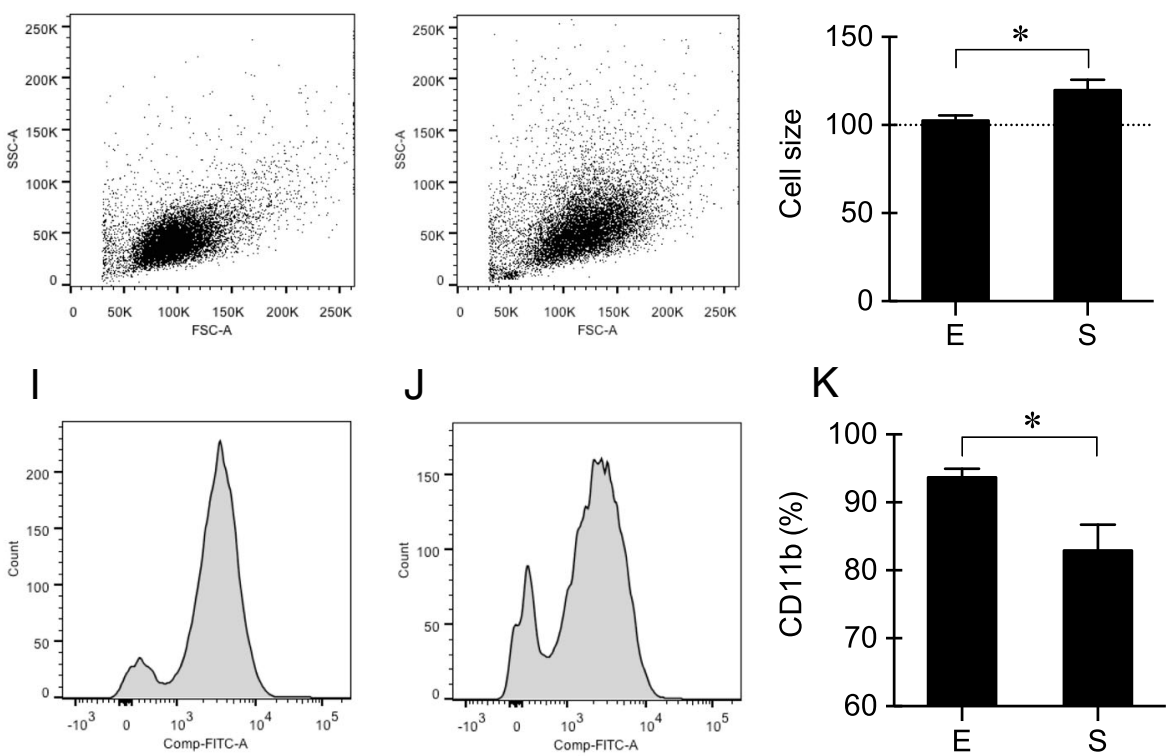

\section{K}

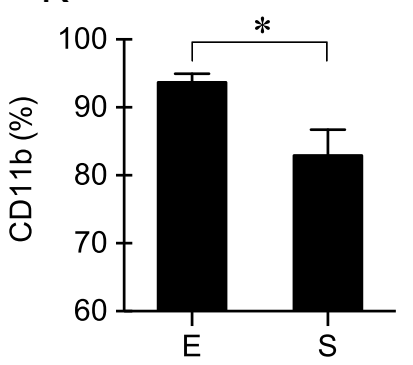

L

M
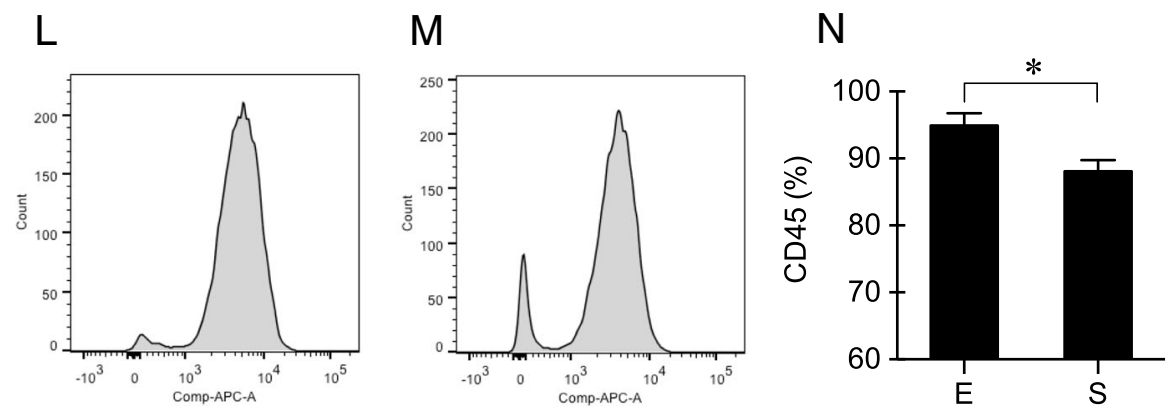

Fig. 2 Morphology, yield, and purity of primary cultured microglia obtained using mild trypsinization (E) or shaking (S). a, b Morphology of primary microglia isolated using mild trypsinization (a) or shaking (b). Scale bar $=50 \mu \mathrm{m}$. c, d Iba-1 immunostaining of primary microglia isolated using mild trypsinization (c) or shaking (d). Scale bar $=50 \mu \mathrm{m}$. e The yield of microglia cultures. $n=5$. $\mathbf{f}-\mathbf{h}$ Size of primary microglia isolated using mild trypsinization (f) or shaking ( $\mathbf{g}) . n=5$. $\mathbf{i}-\mathbf{n}$ Purity of primary microglia isolated using mild trypsinization (i, $\mathbf{l})$ or shaking $(\mathbf{j}, \mathbf{m})$ was determined by flow cytometry with CD11b (i-k) or CD45 (I-n) staining. $n=5 .{ }^{*} p<0.05$ 
Baseline gene expression, cytokine production, and phagocytic function

Because the morphology of microglia appeared to be different in the two preparation methods, we next asked whether these cells might also demonstrate different phenotypes. A representative panel of genes was examined to assess various activation states-iNOS, CD86, CD206, arginase 1, CX3CR1, TLR2, and CCR2. Baseline expression of these selected genes were significantly different in microglia obtained by shaking versus mild trypsinization ( $p=0.003,0.000$, and 0.036 for methods, genes, and methods"genes, respectively, two-way ANOVA) (Fig. 3a).

Microglia are known to influence adjacent cells via the release of extracellular signals. Therefore, we used ELISA to assess key cytokines (TNF $\alpha$, IL-1 $\beta$, and IL-10) and the major microglial growth factor IGF-1 (Fig. 3b). Baseline levels of IL-1 $\beta$ ( $p=0.122, t$ test $)$ and IGF-1 $(p=0.032, t$ test) were about 2 -fold higher in conditioned media from cultured microglia isolated by shaking compared with mild trypsinization. Even larger differences were observed for IL-10 (9-fold, $p=0.048, t$ test) and TNF $\alpha$ (24-fold, $p=0.020, t$ test), again with significantly higher levels from microglia isolated using shaking than using mild trypsinization.

In general, these differences in gene expression and cytokine release suggested that microglia isolated with shaking may be "more activated" compared with those obtained via mild trypsinization. CD68 is considered to be a general marker of activated microglia. Flow cytometry showed that the percentage of CD68-positive cells was slightly higher in microglia isolated using shaking $(39.14 \pm 6.94 \%)$ than using mild trypsinization (31.58 $\pm 7.80 \%$ ), but there was no significant difference between the two methods ( $p=0.185, t$ test) (Fig. 3c-e). However, an in vitro assay demonstrated that phagocytic capacity was significantly enhanced by about

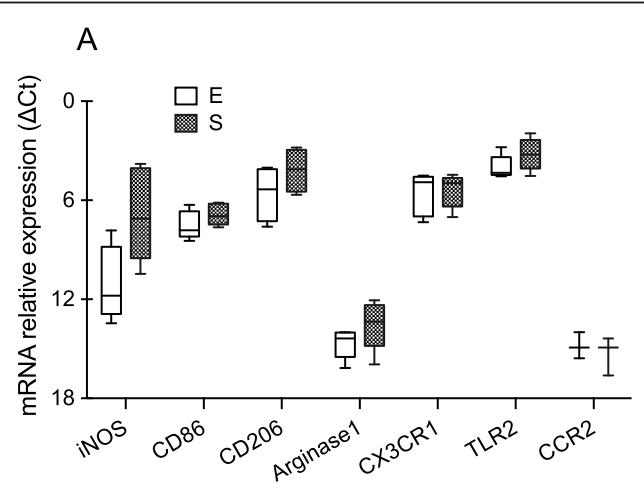

C
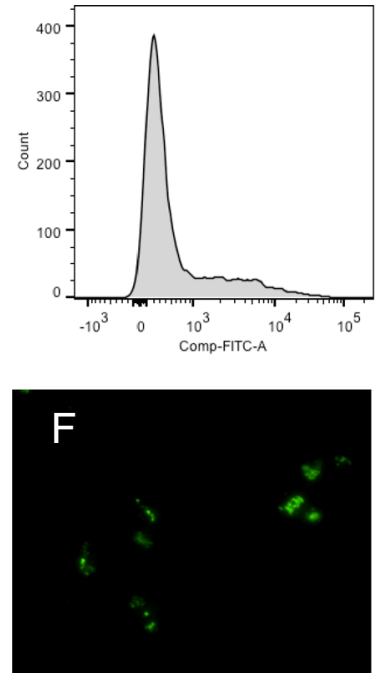

$\mathrm{D}$
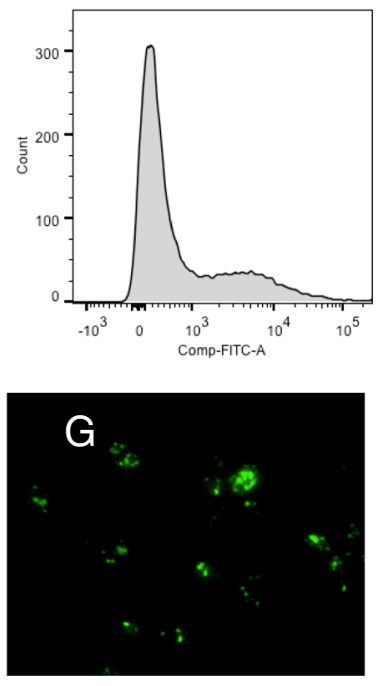
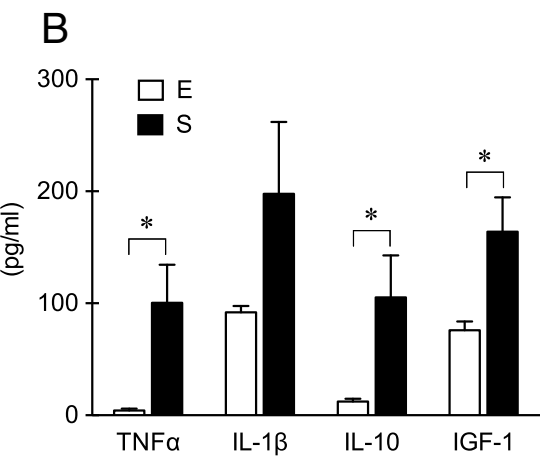

$\mathrm{E}$
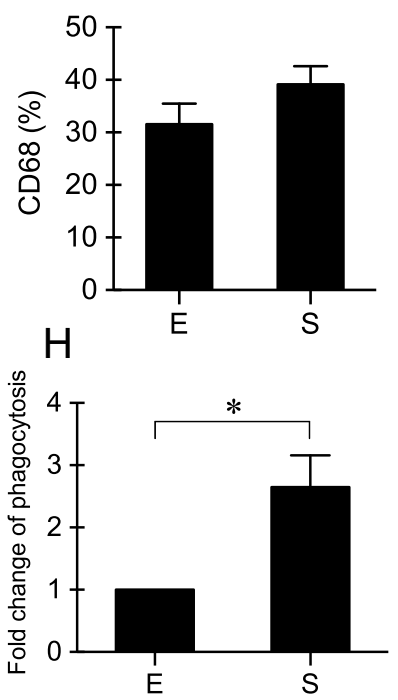

Fig. 3 Baseline levels of gene expression, cytokine release, and phagocytosis of primary cultured microglia isolated using mild trypsinization (E) or shaking (S). a Baseline levels of gene expression. $n=3-6$. b Baseline levels of TNFa, IL-1 $\beta$, IL-10, and IGF-1 in microglial-conditioned media. $n=4$. c-e Flow cytometry showed CD68-positive cells of primary microglia isolated using mild trypsinization (c) or shaking (d). $n=5$. $\mathbf{f}-\mathbf{h}$ Phagocytosis of primary microglia isolated using mild trypsinization (f) or shaking (g). $n=4{ }^{*} p<0.05$ 
2.5-fold in microglia by shaking versus mild trypsinization ( $p=0.018, t$ test) (Fig. $3 \mathrm{f}-\mathrm{h})$.

\section{Comparative response to stimulation}

Next, we tested the response of microglia isolated by these two methods to three typical stimuli that were commonly used to activate microglia into different phenotypes, i.e., IL-4, LPS, and IFNy. After treatment with IL-4 (Fig. 4a) or LPS (Fig. 4b) for $8 \mathrm{~h}$, the pattern of gene expression response (iNOS, CD86, CD206, arginase 1,
CX3CR1, TLR2, and CCR2) appeared to be generally similar (for IL-4 treatment, $p=0.881,0.000$, and 0.408 for methods, genes, and methods"genes, respectively; for LPS treatment, $p=0.962,0.000$, and 0.430 for methods, genes, and methods"genes, respectively, twoway ANOVA). For example, both IL-4 and LPS treatment decreased CX3CR1 expression. IL-4 treatment upregulated the expression of M2-like phenotype marker CD206, whereas LPS treatment upregulated the expression of M1like phenotype marker iNOS and downregulated CD206.

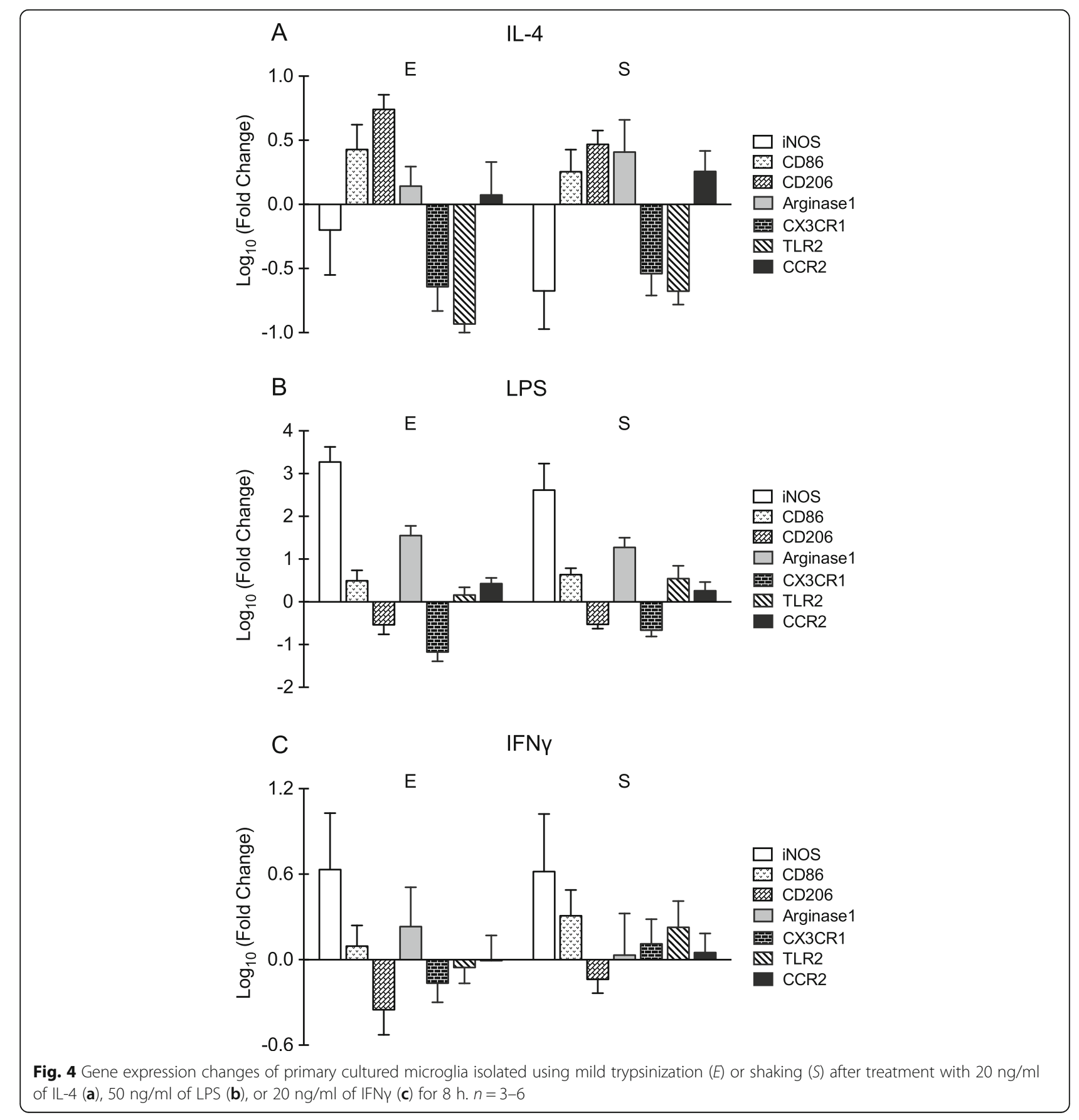


The responses to 8-h IFN $\gamma$ treatments were slightly more variable but once again, overall patterns were similar ( $p=0.333,0.023$, and 0.916 for methods, genes, and methods*genes, respectively, two-way ANOVA). IFN $\gamma$ increased the expression level of iNOS and decreased the level of CD206 in microglia from both shaking and trypsinization groups (Fig. 4c). Consistent with these gene expression findings, morphological changes also appeared to be mostly the same in both groups after various treatments for $24 \mathrm{~h}(20 \mathrm{ng} / \mathrm{ml}$ of IL-4, $50 \mathrm{ng} / \mathrm{ml}$ of LPS, or $20 \mathrm{ng} / \mathrm{ml}$ of IFNY) (Fig. 5a-h).

For further comparisons, the conditioned media after $24 \mathrm{~h}$ treatment with the IL-4, LPS, or IFNy stimuli were collected to measure the levels of secreted cytokines and growth factors. Consistent with the baseline data described above, pre-stimulation levels of TNF $\alpha$, IL-1 $\beta$, IL-10, and IGF-1 were significantly higher in conditioned media of microglia isolated using shaking compared with trypsinization. Stimulation with IL-4, LPS, or IFNy induced distinct responses (Fig. 5i-l, one-way ANOVA). LPS significantly affected TNF $\alpha$, IL-1 $\beta$, and IL-10. IL-4 significantly increased the production of IGF-1, but it had no effect on IL-10, TNF $\alpha$, and IL-1 $\beta$. IFN $\gamma$ had little effects. The direction of the response (increase or decrease) was mostly similar in microglia isolated with both methods (Fig. 5i-l). For example, in microglia isolated from either mild trypsinization or shaking, IL-4 treatment increased IGF-1 release, and LPS treatment increased the generation of TNF $\alpha$ and IL$1 \beta$. However, there were some key differences in the extent of response in some cases. To assess differences of the

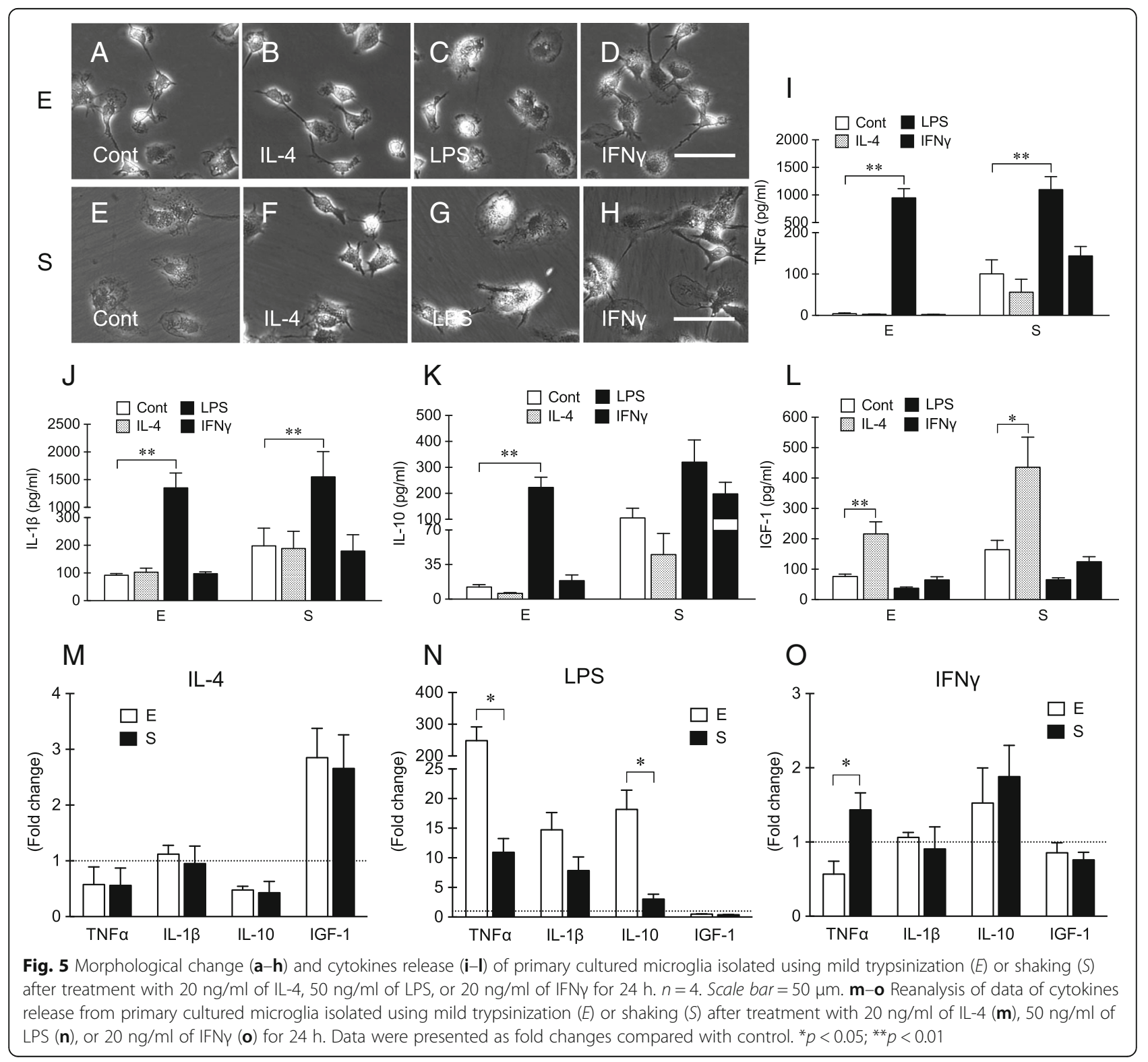


extent of response, we recalculated the data as fold change versus control in Fig. $5 \mathrm{~m}-\mathrm{O}$. Compared with the baseline release, LPS treatment induced significantly higher levels of TNF $\alpha$ and IL-10 in microglia obtained by mild trypsinization than by shaking (Fig. $5 n$ ). For IFN $\gamma$ stimulation, TNF $\alpha$ release was significantly elevated in microglia isolated using shaking, but not in microglia isolated using mild trypsinization (Fig. 5o).

\section{Discussion}

Microglia serve as critical sensors, effectors, and regulators for CNS homeostasis during development and in health and disease [23-25]. Even in healthy brain, microglia are not functionally silent cells. They are highly active, extending and retracting motile processes through which they survey their microenvironment and interact dynamically with surrounding cells [24, 26-28]. Accumulating knowledge now suggest that microglia have multifactorial effects far beyond their traditional roles in immunity [29]. Microglia remove apoptotic neurons, both during CNS development and in adult brains [30-34]. Microglia modulate neurogenesis and promote wiring during embryogenesis and adulthood [35-38]. Microglia take part in synaptic pruning and remodeling in the brain [39-42]. Any insult to the CNS, including infection, trauma, or metabolic dysfunction, causes microglial activation. Upon activation, microglia undergo morphological and functional changes [2, 43]. Microglia can produce numerous mediators including cytokines (both proinflammatory and anti-inflammatory), chemokines, growth factors, and neurotrophins. Microglia can also be phagocytic and generate reactive oxygen and nitrogen species. Therefore, investigations into microglial mechanisms are critically important for a wide spectrum of neuroscience.

Primary cultures comprise a vital in vitro tool for studying microglia. A number of protocols were available for culturing primary microglial cells from neonatal rodent brains. Historically, rodent microglia are isolated using the shaking method [21, 22]. In 2003, another method was introduced that involved mild trypsinization [19]. Although some new methods have been demonstrated to achieve high-yield isolation of microglial cells from postnatal and adult brains [44, 45], shaking remains the most commonly used approach to date (see Fig. 1). Because microglia are highly sensitive and reactive cells, it is possible that different preparation protocols may result in slightly different phenotypes of microglial cells, which in turn can potentially influence experimental outcomes. Therefore, the purpose of the present study was to directly compare key features of microglia isolated using these two methods. Compared to the shaking method, mild trypsinization method to separate microglia from mixed glial cultures generated a higher yield as well as purity. More important is that the shaking method appeared slightly activate microglial cells even under normal condition. Microglial activation is not univalent or bivalent. The concept of classically activated (M1-like) to alternatively activated (M2-like) is derived from the studies of macrophages. Microglia differ from macrophages that reside in other tissues based on their cell-specific gene expression signatures, distinct ontogeny, and differential functions [23, 46-48]. Depending on the stimulus and context, activated microglia exhibit a wide spectrum of phenotypic and functional diversity, not only M1- or M2-like. The so-called M1- or M2-like markers are also not always absolutely limited to one microglial phenotype. Microglia isolated by shaking showed "ameboid" morphology. Compared with mild trypsinization, the baseline expression of microglial activation markers (iNOS, CD86, CD206, and arginase 1) and the baseline release of cytokines (TNF $\alpha$, IL$1 \beta$, IL-10, and IGF-1) were significantly higher in microglia isolated by shaking. Microglia isolated using shaking also showed enhanced phagocytosis when compared with the microglia isolated using mild trypsinization. These findings further confirm that primary cultured microglia are extremely sensitive to stimuli, and responses are sometimes dependent on isolation protocols. Hence, caution may be required in choosing methods and interpreting data. For studies that focus on baseline microglial physiology, it may be better to select methods that minimize inadvertent activation. Caveats must also be acknowledged when investigating pathways by probing with a recombinant protein, especially if the purity is lower and the level of endotoxin in the recombinant protein is higher.

In our study, we documented the changes of gene expression (iNOS, CD86, CD206, arginase 1, CX3CR1, TLR2, and CCR2) and cytokine release (TNF $\alpha$, IL-1 $\beta$, IL-10, and IGF-1) in microglia obtained from these two methods in response to various typical stimuli that may induce M1-like (LPS or IFNY) or M2-like (IL-4) phenotypes. The microglia obtained by either mild trypsinization or shaking were fully functional and generally responded to different treatments as expected. However, the extent of responses appeared to be different under some conditions. Even though both methods were feasible for evaluating functional responses of microglia in vitro, opposite conclusions may arise if the extent of response was critical for a particular study. For example, to evaluate the beneficial or harmful effects of activated microglia, the levels of TNF $\alpha$ released from activated microglia may be a key. Different concentrations of TNF $\alpha$ offer distinct receptor selectivity [49]. Higher levels of TNF $\alpha$ may induce neuronal death and increase glutamate release, but lower levels of TNF $\alpha$ may not $[50,51]$. In this scenario, the different responses of TNF $\alpha$ to LPS or IL-4 stimulation in microglia isolated by shaking or trypsinization may affect results and conclusions. 
There are a few caveats. First, the purity of cultured cells is one of the critical factors that would influence the experimental results. Unfortunately, it is almost impossible to obtain $100 \%$ pure cells in any primary culture. In our primary microglial cultures, other types of brain cells, including neurons, astrocytes, and oligodendrocytes, might exist. However, the differences in purity might not be a major contributor to the differential inflammatory responses or baseline immune mediator expression in this study because the genes and cytokines we measured are mainly expressed by microglia, rather than neurons, astrocytes, or oligodendrocytes. Actually, one potential advantage of using mild trypsinization to culture microglia may be because this method provides about 95\% pure microglial cells. Nevertheless, if these cultures were further passaged, other cells may still persist and the proportion of various cell types might be changed. This is an important caveat. Second, the speed and duration of shaking might be important to influence the characteristics of microglia isolated by shaking. Shaking speed and duration to isolate microglia from mixed cultures are variable in the literatures. In the present study, we used shaking speed and duration that were typically used in our lab. Compared with the literature, $220 \mathrm{rpm}$ is a commonly used speed [52, 53]. Third, since microglia are very sensitive to even tiny stimulations, it is possible that the phenotype of microglia would be switched if the microglia were allowed to recuperate for a longer time after being isolated from the mixed cultures, such as $48 \mathrm{~h}$ instead of overnight. Future studies to assess various time points after the shaking method would be beneficial. Fourth, although we showed that the yield was higher using the mild trypsinization method than the shaking method in the present study, we acknowledge that we do not know what causes the difference of the yield in the two methods. We plated cells from one neonatal brain into one 6-well plate to make sure that the initial culture conditions are same in both methods. Before taking the photos to compare the yield, we cultured the microglial cells for another $24 \mathrm{~h}$ after isolating them from the mixed cultures using either shaking or mild trypsinization. However, potential differences in microglial mechanisms of response to enzyme versus mechanical manipulations remain to be elucidated.

Recently, other studies have also raised the possibility that the shaking method might inadvertently activate microglia, and immunomagnetic microbead methods have been proposed as an alternative way for in vitro isolation $[52,54,55]$. Here, we showed that the mild trypsinization method resulted in similar purity as the magnetic microbead method. The purity of mild trypsinization method used in the present study is about $95 \%$. The purity of magnetic microbead method is about 95$97 \%$ according to the literature [52, 54, 55]. Compared with magnetic sorting, mild trypsinization might also be cheaper (no need for extra reagents and equipment) and simpler (no extra procedures).

\section{Conclusions}

In summary, mild trypsinization may be a reliable method to isolate microglia from mixed glial cultures with increased yield and purity, and microglial purified by mild trypsinization may be closer to their "resting" state. The immune state of microglia was influenced by the method of purification, and the culture method should be carefully considered in in vitro research.

\begin{abstract}
Abbreviations
B2M: ß2-Microglobulin; CCR2: C-C chemokine receptor 2; CNS: Central nervous system; CX3CR1: CX3C chemokine receptor 1; E: Mild trypsinization; FBS: Fetal bovine serum; IFNY: Interferon-y; IGF-1: Insulin-like growth factor-1; IL: Interleukin; iNOS: Inducible nitric oxide synthase; LPS: Lipopolysaccharides; S: Shaking; TLR2: Toll-like receptor 2; TNFa: Tumor necrosis factor a
\end{abstract}

\section{Acknowledgements}

Not applicable.

\section{Funding}

This work was supported in part by grants from the $\mathrm{NIH}$ and the Rappaport Foundation.

\section{Availability of data and materials}

All data generated or analyzed during this study are included in this published article.

\section{Authors' contributions}

$E L$ and $C X$ designed the study. $L L$ and $C X$ performed the experiments. RD did the PubMed search. CX and XW analyzed the data. EL and CX wrote the paper. All authors read and approved the final manuscript.

\section{Competing interests}

The authors declare that they have no competing interests.

\section{Consent for publication}

Not applicable.

\section{Ethics approval}

All experiments were approved by the Massachusetts General Hospital Institutional Animal Care and Use Committee (IACUC) following standard protocols according to the NIH Guidelines for the Care and Use of Animals in Biomedical Research.

\section{Publisher's Note}

Springer Nature remains neutral with regard to jurisdictional claims in published maps and institutional affiliations.

\section{Author details}

${ }^{1}$ School of Pharmaceutical Sciences, Wenzhou Medical University, Wenzhou, Zhejiang 325035, China. ${ }^{2}$ Neuroprotection Research Laboratory, Departments of Radiology and Neurology, Massachusetts General Hospital, Harvard Medical School, MGH East 149-2401, Charlestown, MA 02129, USA.

Received: 12 February 2017 Accepted: 1 May 2017

Published online: 08 May 2017

\section{References}

1. Parkhurst CN, Gan WB. Microglia dynamics and function in the CNS. Curr Opin Neurobiol. 2010;20(5):595-600.

2. Ransohoff RM, Perry VH. Microglial physiology: unique stimuli, specialized responses. Annu Rev Immunol. 2009;27:119-45. 
3. Polazzi E, Monti B. Microglia and neuroprotection: from in vitro studies to therapeutic applications. Prog Neurobiol. 2010;92(3):293-315.

4. Benoit M, Desnues B, Mege JL. Macrophage polarization in bacterial infections. J Immunol. 2008;181(6):3733-9.

5. Geissmann F, Auffray C, Palframan R, Wirrig C, Ciocca A, Campisi L, et al. Blood monocytes: distinct subsets, how they relate to dendritic cells, and their possible roles in the regulation of T-cell responses. Immunol Cell Biol. 2008;86(5):398-408.

6. Mantovani A, Sica A, Sozzani S, Allavena P, Vecchi A, Locati M. The chemokine system in diverse forms of macrophage activation and polarization. Trends Immunol. 2004;25(12):677-86.

7. Michelucci A, Heurtaux T, Grandbarbe L, Morga E, Heuschling P. Characterization of the microglial phenotype under specific proinflammatory and anti-inflammatory conditions: effects of oligomeric and fibrillar amyloid-beta. J Neuroimmunol. 2009;210(1-2):3-12.

8. Giunti D, Parodi B, Cordano C, Uccelli A, Kerlero de Rosbo N. Can we switch microglia's phenotype to foster neuroprotection? Focus on multiple sclerosis. Immunology. 2014;141(3):328-39.

9. Mosser DM, Edwards JP. Exploring the full spectrum of macrophage activation. Nat Rev Immunol. 2008;8(12):958-69.

10. Gordon S. The macrophage: past, present and future. Eur J Immunol. 2007; 37 Suppl 1:S9-17.

11. O'Shea JJ, Murray PJ. Cytokine signaling modules in inflammatory responses. Immunity. 2008;28(4):477-87.

12. Geissmann F, Gordon S, Hume DA, Mowat AM, Randolph GJ. Unravelling mononuclear phagocyte heterogeneity. Nat Rev Immunol. 2010;10(6):453-60

13. Mantovani A, Sica A, Locati M. Macrophage polarization comes of age. Immunity. 2005;23(4):344-6.

14. Gordon S. Alternative activation of macrophages. Nat Rev Immunol. 2003; 3(1):23-35.

15. Popovich PG, Longbrake EE. Can the immune system be harnessed to repair the CNS? Nat Rev Neurosci. 2008;9(6):481-93.

16. Gratchev A, Guillot P, Hakiy N, Politz O, Orfanos CE, Schledzewski K, et al. Alternatively activated macrophages differentially express fibronectin and its splice variants and the extracellular matrix protein betalG-H3. Scand J Immunol. 2001;53(4):386-92.

17. Arnold L, Henry A, Poron F, Baba-Amer Y, van Rooijen N, Plonquet A, et al. Inflammatory monocytes recruited after skeletal muscle injury switch into antiinflammatory macrophages to support myogenesis. J Exp Med. 2007; 204(5):1057-69.

18. Eggen BJ, Raj D, Hanisch UK, Boddeke HW. Microglial phenotype and adaptation. J Neuroimmune Pharmacol. 2013;8(4):807-23.

19. Saura J, Tusell JM, Serratosa J. High-yield isolation of murine microglia by mild trypsinization. Glia. 2003;44(3):183-9.

20. Xing C, Wang X, Cheng C, Montaner J, Mandeville E, Leung W, et al. Neuronal production of lipocalin-2 as a help-me signal for glial activation. Stroke. 2014:45(7):2085-92.

21. Giulian D, Baker TJ. Characterization of ameboid microglia isolated from developing mammalian brain. J Neurosci. 1986;6(8):2163-78.

22. Frei K, Bodmer S, Schwerdel C, Fontana A. Astrocyte-derived interleukin 3 as a growth factor for microglia cells and peritoneal macrophages. J Immunol. 1986;137(11):3521-7.

23. Prinz M, Priller J. Microglia and brain macrophages in the molecular age: from origin to neuropsychiatric disease. Nat Rev Neurosci. 2014;15(5):300-12.

24. Hanisch UK, Kettenmann $\mathrm{H}$. Microglia: active sensor and versatile effector cells in the normal and pathologic brain. Nat Neurosci. 2007;10(11):1387-94.

25. Kreutzberg GW. Microglia: a sensor for pathological events in the CNS. Trends Neurosci. 1996;19(8):312-8

26. Davalos D, Grutzendler J, Yang G, Kim JV, Zuo Y, Jung S, et al. ATP mediates rapid microglial response to local brain injury in vivo. Nat Neurosci. 2005; 8(6):752-8.

27. Nimmerjahn A, Kirchhoff F, Helmchen F. Resting microglial cells are highly dynamic surveillants of brain parenchyma in vivo. Science. 2005; 308(5726):1314-8

28. Kettenmann $H$, Verkhratsky A. Neuroglia: the 150 years after. Trends Neurosci. 2008;31(12):653-9.

29. Graeber MB, Streit WJ. Microglia: biology and pathology. Acta Neuropathol. 2010;119(1):89-105.

30. Perry VH, Hume DA, Gordon S. Immunohistochemical localization of macrophages and microglia in the adult and developing mouse brain. Neuroscience. 1985;15(2):313-26.
31. Ashwell K. Microglia and cell death in the developing mouse cerebellum. Brain Res Dev Brain Res. 1990;55(2):219-30.

32. Caldero J, Brunet N, Ciutat D, Hereu M, Esquerda JE. Development of microglia in the chick embryo spinal cord: implications in the regulation of motoneuronal survival and death. J Neurosci Res. 2009;87(11):2447-66.

33. Sierra A, Encinas JM, Deudero JJ, Chancey JH, Enikolopov G, OverstreetWadiche LS, et al. Microglia shape adult hippocampal neurogenesis through apoptosis-coupled phagocytosis. Cell Stem Cell. 2010;7(4):483-95.

34. Neumann $\mathrm{H}$, Kotter MR, Franklin RJ. Debris clearance by microglia: an essential link between degeneration and regeneration. Brain. 2009;132(Pt 2):288-95.

35. Ueno M, Fujita Y, Tanaka T, Nakamura Y, Kikuta J, Ishii M, et al. Layer V cortical neurons require microglial support for survival during postnatal development. Nat Neurosci. 2013;16(5):543-51.

36. Shigemoto-Mogami Y, Hoshikawa K, Goldman JE, Sekino Y, Sato K. Microglia enhance neurogenesis and oligodendrogenesis in the early postnatal subventricular zone. J Neurosci. 2014;34(6):2231-43.

37. Squarzoni $P$, Oller $G$, Hoeffel G, Pont-Lezica L, Rostaing P, Low D, et al. Microglia modulate wiring of the embryonic forebrain. Cell Rep. 2014; 8(5):1271-9.

38. Cunningham CL, Martinez-Cerdeno V, Noctor SC. Microglia regulate the number of neural precursor cells in the developing cerebral cortex. J Neurosci. 2013:33(10):4216-33.

39. Wake H, Moorhouse AJ, Jinno S, Kohsaka S, Nabekura J. Resting microglia directly monitor the functional state of synapses in vivo and determine the fate of ischemic terminals. J Neurosci. 2009;29(13):3974-80.

40. Paolicelli RC, Bolasco G, Pagani F, Maggi L, Scianni M, Panzanelli P, et al. Synaptic pruning by microglia is necessary for normal brain development. Science. 2011;333(6048):1456-8.

41. Tremblay ME, Lowery RL, Majewska AK. Microglial interactions with synapses are modulated by visual experience. PLoS Biol. 2010;8(11), e1000527.

42. Holtmaat A, Svoboda K. Experience-dependent structural synaptic plasticity in the mammalian brain. Nat Rev Neurosci. 2009;10(9):647-58.

43. Ransohoff RM, Cardona AE. The myeloid cells of the central nervous system parenchyma. Nature. 2010;468(7321):253-62.

44. Masuch A, van der Pijl R, Funer L, Wolf Y, Eggen B, Boddeke E, et al. Microglia replenished OHSC: a culture system to study in vivo like adult microglia. Glia. 2016;64(8):1285-97.

45. Sepulveda-Diaz JE, Ouidja MO, Socias SB, Hamadat S, Guerreiro S, RaismanVozari $R$, et al. A simplified approach for efficient isolation of functional microglial cells: application for modeling neuroinflammatory responses in vitro. Glia. 2016;64(11):1912-24.

46. Gautier EL, Shay T, Miller J, Greter M, Jakubzick C, Ivanov S, et al. Gene-expression profiles and transcriptional regulatory pathways that underlie the identity and diversity of mouse tissue macrophages. Nat Immunol. 2012;13(11):1118-28.

47. Ginhoux F, Greter M, Leboeuf M, Nandi S, See P, Gokhan S, et al. Fate mapping analysis reveals that adult microglia derive from primitive macrophages. Science. 2010;330(6005):841-5.

48. Kierdorf K, Erny D, Goldmann T, Sander V, Schulz C, Perdiguero EG, et al. Microglia emerge from erythromyeloid precursors via Pu.1- and Iff8dependent pathways. Nat Neurosci. 2013;16(3):273-80.

49. Fischer R, Maier O, Siegemund M, Wajant H, Scheurich P, Pfizenmaier K. A TNF receptor 2 selective agonist rescues human neurons from oxidative stress-induced cell death. PLoS One. 2011;6(11), e27621.

50. Martinez TN, Chen X, Bandyopadhyay S, Merrill AH, Tansey MG. Ceramide sphingolipid signaling mediates tumor necrosis factor (TNF)-dependent toxicity via caspase signaling in dopaminergic neurons. Mol Neurodegener. 2012;7:45.

51. Chen CJ, Ou YC, Chang CY, Pan HC, Liao SL, Chen SY, et al. Glutamate released by Japanese encephalitis virus-infected microglia involves TNFalpha signaling and contributes to neuronal death. Glia. 2012;60(3):487-501.

52. Ju L, Zeng $H$, Chen $Y$, Wu Y, Wang $B, X u Q$. Dual polarization of microglia isolated from mixed glial cell cultures. J Neurosci Res. 2015;93(9):1345-52.

53. Beutner C, Linnartz-Gerlach B, Schmidt SV, Beyer M, Mallmann MR, Staratschek-Jox A, et al. Unique transcriptome signature of mouse microglia. Glia. 2013;61(9):1429-42.

54. Jose S, Tan SW, Tong CK, Vidyadaran S. Isolation and characterization of primary microglia from post-natal murine brain tissues: a comparison of two methods. Cell Biol Int. 2015;39(12):1355-63.

55. Gordon R, Hogan CE, Neal ML, Anantharam V, Kanthasamy AG, Kanthasamy A. A simple magnetic separation method for high-yield isolation of pure primary microglia. J Neurosci Methods. 2011;194(2):287-96. 